\title{
Biocompatible Graphene Oxide Nanosheets Densely Functionalized with Biologically-Active Molecules for Biosensing Applications
}

Benjamin A. E. Lehner, ${ }^{1}$ Dominik Benz, ${ }^{2}$ Stanlislav A. Moshkalev, ${ }^{3}$ Anne S. Meyer,${ }^{4}$ Monica A. Cotta, ${ }^{5}$ and Richard Janissen ${ }^{6 *}$

${ }^{1}$ Kavli Institute of Nanoscience, Delft University of Technology, Delft, 2629HZ, The Netherlands

${ }^{2}$ Chemical Engineering, Delft University of Technology, Delft, 2629HZ, The Netherlands

${ }^{3}$ Center of Semiconductor Components and Nanotechnologies, University of Campinas, Campinas, Sao Paulo, 13083-870, Brazil

${ }^{4}$ Department of Biology, University of Rochester, Rochester, NY 14627, USA ${ }^{5}$ Laboratory of Nano and Biosystems, Department of Applied Physics, University of Campinas, Campinas, Sao Paulo, 13083-859, Brazil

${ }^{1}$ Kavli Institute of Nanoscience, Delft University of Technology, Delft, 2629HZ, The Netherlands

*Corresponding author: r.janissen@tudelft.nl 


\section{Supplementary Figures}
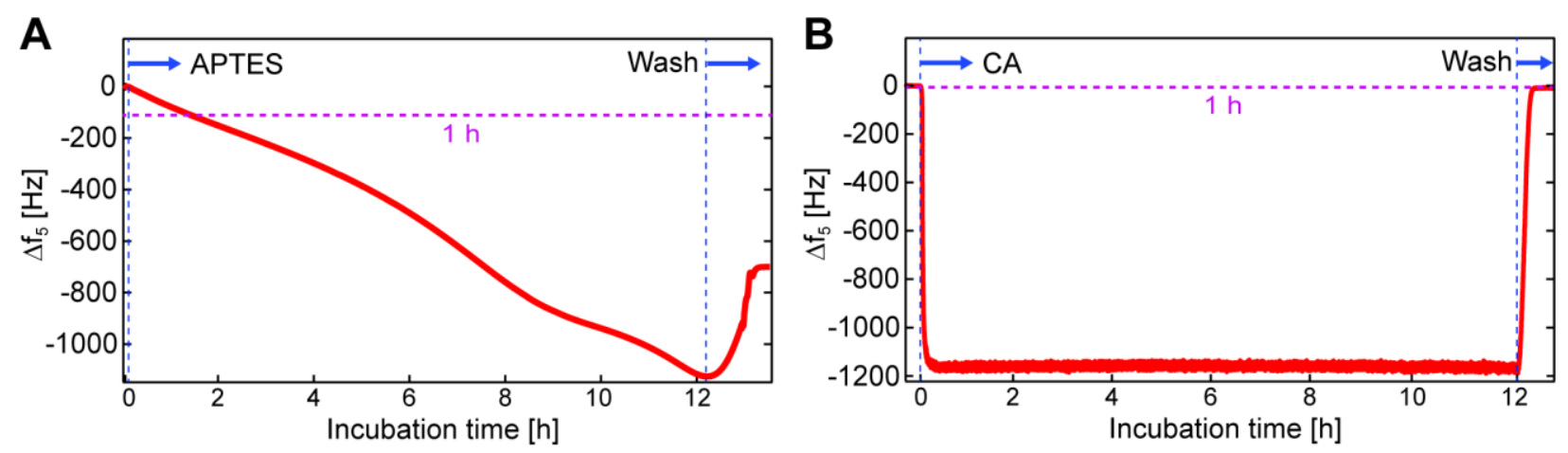

Figure S1. ATPES and CA surface deposition after $12 \mathrm{~h}$. Surface deposition of (A) APTES and (B) CA over 12 hours measured via QCM-D. Averaged ( $\pm \mathrm{SD})$, normalized frequency shifts, $\Delta f=$ $\Delta f_{i} / i$ for the overtones $i=\{5,7,9\}$ are presented in both plots (red). Resulting areal molar density and estimated molecular layers for APTES and CA deposition remaining bound after washing are quantified in Figure 1. For comparison of the effect of chemical deposition times, the average $\Delta f$ resulting from $1 \mathrm{~h}$ surface deposition (Figure 1) of both crosslinker is depicted in the plots (magenta dashed lines). Related to Figure 1. 

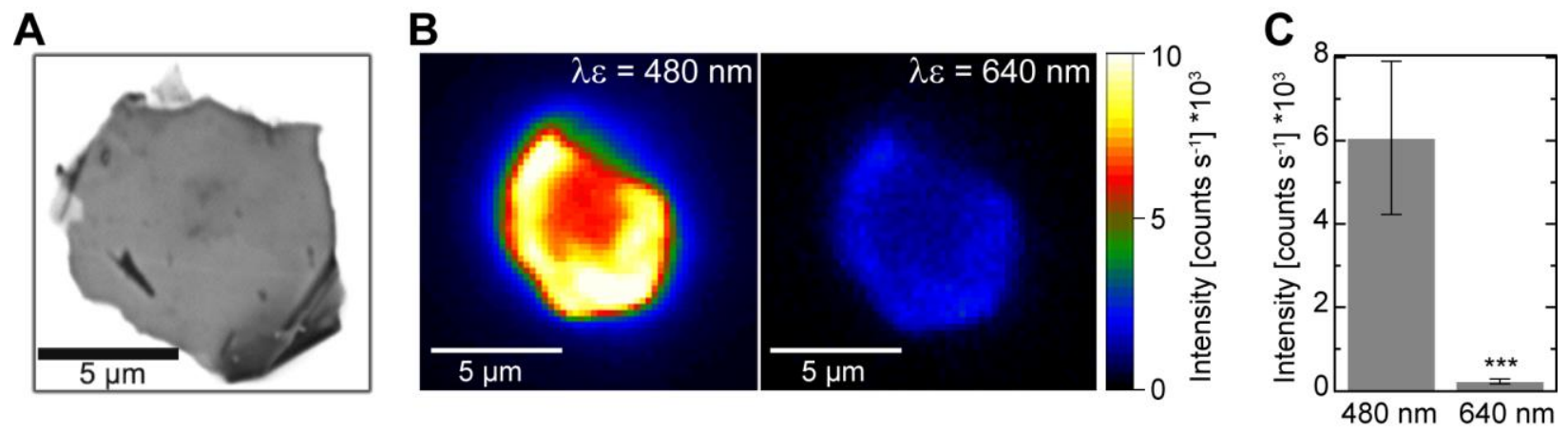

Figure S2. Autofluorescence of exfoliated graphene oxide (GO). (A) SEM micrograph of exfoliated GO tested for autofluorescence. (B) Example false-color fluorescence images of a GO flake show different autofluorescence intensity in the green and red emission wavelength regimes

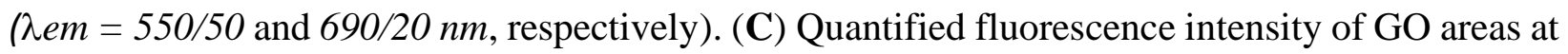
different wavelengths exhibits significantly lower autofluorescence in the red wavelength regime. Statistical analysis was performed using an unpaired, two-tailed t-test (significance level $\mathrm{p}$ : ${ }^{* *} \leq$ 0.001). Related to Figures 1 and 4. 

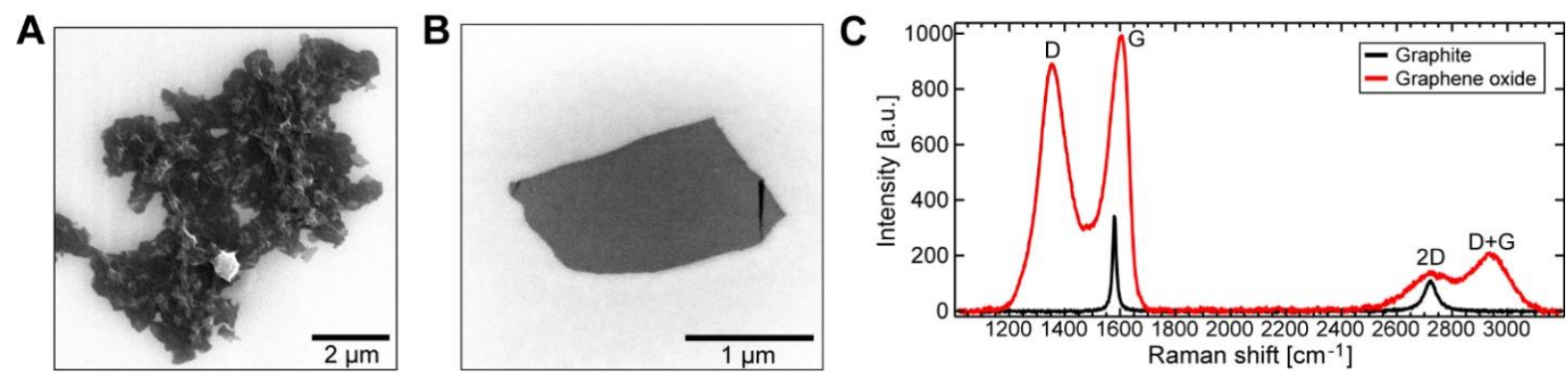

Figure S3. Scanning electron microscopy (SEM) and Raman spectroscopy of primary graphite material and exfoliated graphene oxide (GO). (A) SEM micrograph of a graphite flake used as primary material before exfoliation and oxidation. (B) SEM micrograph of an exfoliated and reduced GO flake on a silicon dioxide surface. (C) Raman spectrometry of primary graphite (black) material and GO sheets (red). The D peak appears after oxidation due to out-ofplane vibrations caused by oxygen atoms. All peaks in $\mathrm{GO}$ are broader than in graphite due to the increased amount of induced structural defects. Related to Figure 2. 

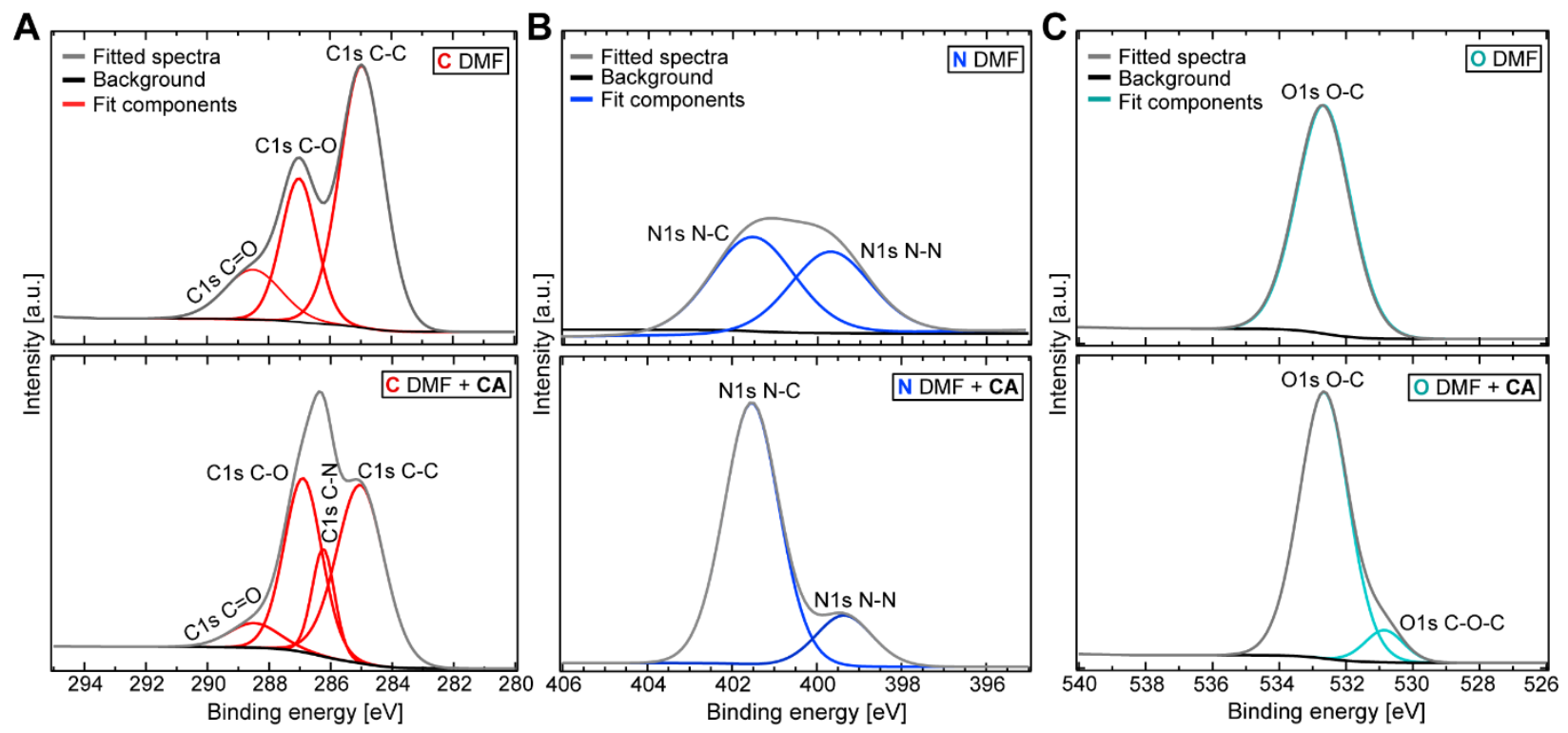

Figure S4. HR-XPS analysis of CA-functionalized of GO in DMF. (A) Carbon, (B) nitrogen, and (C) oxygen high-resolution (HR) XPS spectra before (top panels) and after (bottom panels) addition of CA. A carbon-nitrogen binding exhibited the best fit for the XPS spectrum with CA treatment (bottom panels), reflecting a 2-fold increase in peak intensity. Grey lines represent deconvoluted multi-peak fittings. Related to Figure 3. 